ISSN 1678-3921

Journal homepage: www.embrapa.br/pab

For manuscript submission and journal contents, access: www.scielo.br/pab
Allan Ricardo Domingues ${ }^{(1)}(\mathbb{D}$,

Ciro Daniel Marques Marcolini(2) (D), Carlos Henrique da Silva Gonçalves ${ }^{(1)}$ (iD, Carlos Eduardo Alves da Silva(2) (iD, Sergio Ruffo Roberto ${ }^{(1 \otimes)}(\mathbb{D})$ and Eduardo Fermino Carlos ${ }^{(2)}$ (D)

(1) Universidade Estadual de Londrina, Rodovia Celso Garcia Cid, PR 445, Km 380, Caixa Postal 10.011, CEP 86057-970 Londrina, PR, Brazil. E-mail: allanrdomingues@gmail.com carloshg@itaipu.gov.br, sroberto@uel.br

(2) Instituto de Desenvolvimento Rural do Paraná, Rodovia Celso Garcia Cid, Km 375 , CEP 86047-902 Londrina, PR, Brazil. E-mail: ciromarcolini@emater.pr.gov.br carlos.ramed@gmail.com, efcarlos@idr.pr.gov.br

$\bowtie$ Corresponding author

Received

January 22, 2021

Accepted

July 15, 2021

How to cite DOMINGUES, A.R.; MARCOLINI, C.D.M.; GONÇALVES, C.H. da S.; SILVA, C.E.A. da; ROBERTO, S.R.; CARLOS, E.F. Performance of 'Valência' sweet orange grafted on trifoliate orange hybrid rootstocks. Pesquisa Agropecuária Brasileira, v.56, e02403, 2021. DOI: https://doi.org/10.1590/S1678-3921. pab2021.v56.02403.

\section{Performance of 'Valência' sweet orange grafted on trifoliate orange hybrid rootstocks}

Abstract - The objective of this work was to evaluate tree size, production, and fruit quality of 'Valência' sweet orange (Citrus sinensis) grafted on various trifoliate orange rootstocks, in order to select genotypes with a high performance. Twenty rootstock genotypes were evaluated, including trifoliate orange hybrids with mandarin (citrandarins) and with grapefruit (citrumelos), as well as 'Rangpur' lime. The experiment was implemented in the northwestern region of the state of Paraná, Brazil, in a $6.0 \times 2.5 \mathrm{~m}$ spacing, in a sandy soil under subtropical and rainfed conditions. The statistical model used was the randomized complete block design with four replicates and four trees per plot. Tree size and fruit yield and quality were analyzed during three consecutive harvest seasons. Data were subjected to the analysis of variance, and means were grouped by the Scott-Knott test. Principal component analysis and agglomerative hierarchical clustering were also carried out. F.80-18, F.805, and F.80-3 citrumelos and IPEACS-239 citrandarin are adequate rootstock options for 'Valência' sweet orange, with dwarf trees and a high production efficiency. These rootstocks, except F.80-18, are also adequate options to obtain oranges with good industrial properties.

Index terms: Citrus sinensis, Poncirus trifoliata, canopy volume, citrandarin, citrumelo, fruit quality, yield efficiency.

\section{Desempenho da laranjeira 'Valência' enxertada sobre porta-enxertos híbridos de trifoliateiro}

Resumo - O objetivo deste trabalho foi avaliar o tamanho da planta, a produção e a qualidade dos frutos da laranjeira-doce 'Valência' (Citrus sinensis) enxertada sobre diversos porta-enxertos híbridos de trifoliateiro, para selecionar genótipos de alto desempenho. Vinte genótipos de porta-enxertos foram avaliados, tendo incluído híbridos de trifoliateiro com tangerineiras (citrandarins) e com pomeleiros (citrumelos), além do limoeiro 'Cravo'. O experimento foi implantado na região noroeste do estado do Paraná, Brasil, em espaçamento $6.0 \times 2.5 \mathrm{~m}$, em solo arenoso, sob condições de clima subtropical e sem irrigação. O modelo estatístico utilizado foi o delineamento em blocos ao acaso, com quatro repetições e quatro plantas por parcela. O tamanho da planta e a produção e a qualidade dos frutos foram analisados durante três safras consecutivas. Os dados foram submetidos à análise de variância, e as médias foram agrupadas pelo teste de Scott-Knott. Também foram realizados a análise de componentes principais e o agrupamento dos porta-enxertos por meio da classificação hierárquica de clusters. Os citrumeleiros F.80-18, F.80-5 e F.80-3 e o citrandarineiro IPEACS-239 são opções adequadas de porta-enxertos para a laranjeira 'Valência', com plantas anãs e alta eficiência produtiva. Esses porta-enxertos, exceto F.80-18, também são opções adequadas para a obtenção de laranjas com boas propriedades industriais.

Termos para indexação: Citrus sinensis, Poncirus trifoliata, volume de copa, citrandarin, citrumelo, qualidade de frutos, eficiência produtiva. 


\section{Introduction}

'Valência' sweet orange [Citrus sinensis (L.) Osbeck] is one of the most cultivated varieties worldwide, with the characteristics - high fruit production and quality - required by the juice industry and by the fresh fruit market (Saunt, 2000; Bastos et al., 2014). It is graft-compatible with various rootstocks, including 'Rangpur' lime (Citrus limonia Osbeck) and 'Swingle' citrumelo [Citrus paradisi MacFad. $\times$ Poncirus trifoliata (L.) Raf.], the most used in Brazil, as well as with some trifoliate orange hybrids, which are a new generation of rootstocks with potential for use in the citrus industry (Simonetti et al., 2015).

Citrus growers seek rootstocks that induce smaller trees to allow high-density plantings, in order to facilitate management and harvesting, increase yield, and provide a high fruit quality (Carvalho et al., 2019a). In addition, it is important that the rootstocks chosen for each scion provide a high production efficiency, as well as resistance to the main diseases and abiotic factors that affect citrus orchards (Castle et al., 2010; Domingues et al., 2018).

Among the promising genotypes of rootstocks, some hybrids of trifoliate orange stand out, such as citrumelos and citrandarins (Citrus reticulata Blanco $\times$ P. trifoliata) (Donadio et al., 2019; Domingues et al., 2021a).

'Swingle' citrumelo is the main rootstock used in Florida, USA, and one of the most important in Brazil (FDACS, 2018; Carvalho et al., 2019b). This rootstock induces a high orange production and high contents of total soluble solids, besides tolerance to citrus blight, sudden death, tristeza virus, and Phytophthora spp. gummosis (Castle et al., 2010). Considering the importance of this trifoliate hybrid, other rootstocks, designated as F.80 series, obtained from a similar crossing ('Duncan' citrumelo $\times P$. trifoliata), have most of the features of 'Swingle' citrumelo and induce trees with a low vigor and small size (Pompeu Junior \& Blumer, 2011).

'US-852' (C. reticulata 'Changsha' $\times P$. trifoliata 'English Large') and 'US-812' [Citrus sunki (Hayata) hort. ex Tanaka $\times$ P. trifoliata 'Benecke'] citrandarins are commercially grown in the US and induce a high fruit quality and production, as well as less vigorous trees than other rootstocks such as 'Swingle' citrumelo (Vashisth et al., 2020). In Brazil, 'Pêra' sweet orange grafted on 'US-801' (C. reticulata 'Changsha' $\times P$. trifoliata 'English Large') and IPEACS-264 (C. sunki $\times$ P. trifoliata 'English') citrandarins presented high yields (Pompeu Junior \& Blumer, 2014). A similar performance was observed for 'Pêra' on some hybrids of 'Sunki' mandarin $\times P$. trifoliata, which induced a high fruit production and quality, and, in some cases, dwarfing potential (Schinor et al., 2013). However, there is still a lack of information about the performance of these rootstocks for 'Valência' sweet orange grown in the northwest of the state of Paraná, Brazil.

The objective of this work was to evaluate tree size, production, and fruit quality of 'Valência' sweet orange grafted on various trifoliate orange rootstocks, in order to select genotypes with a high performance.

\section{Materials and Methods}

The experiment was conducted at the Experimental Research Station of Cocamar Cooperative in the municipality of Guairaça, located in the northwestern region of the state of Paraná, Brazil (22 $2^{\circ} 56^{\prime} 30^{\prime \prime} \mathrm{S}$, $52^{\circ} 43^{\prime} 48^{\prime \prime} \mathrm{W}$, at $470 \mathrm{~m}$ above sea level), during consecutive harvest seasons. The climate of the region is Cfa, according to Köppen's classification, subtropical with hot summers, infrequent frosts, and concentration of the rainiest period in the summer months, but without a well-defined dry season. The average annual rainfall ranges from 1,400 to 1,600 $\mathrm{mm}$, the average temperature from 22.1 to $23.0^{\circ} \mathrm{C}$, and relative humidity from 65 to $70 \%$ (Nitsche et al., 2019). The soil is medium textured and composed of sand (or sandstone) on horizon A and clay on horizon B, being classified as a Argissolo Vermelho-Amarelo (Santos et al., 2018), i.e., an Oxisol. Additional information about the weather conditions during the trial, including temperature, rainfall, and water balance are available at the website of IDR-Paraná (2021).

Field-ready and grafted 'Valência' sweet orange trees of clone IAC (C. sinensis) (Pratinhas's Citrus Nursery, Paranavaí, PR, Brazil), were planted in April 2013 in a $6.0 \times 2.5 \mathrm{~m}$ spacing, at 667 trees per hectare, under rainfed conditions. The randomized complete block design was used as a statistical model, with four replicates and four trees per plot.

The treatments consisted of 20 rootstock genotypes donated by the Centro de Citricultura Sylvio Moreira, Instituto Agronômico de Campinas (CCSM/IAC), 
Brazil, including hybrids of trifoliate orange ( $P$. trifoliata) with mandarins (citrandarins) and with grapefruit (citrumelos), as well as with 'Rangpur' lime and the following other potential rootstocks: 'US-852' citrandarin; 'US-801' citrandarin; 'US-812' citrandarin; IPEACS-256 citrandarin (C. reticulata 'Cleopatra' $P$. trifoliata $\times$ 'English'); IPEACS-239 citrandarin (C. reticulata 'Cleopatra' $\times P$. trifoliata 'Rubidoux'); IPEACS-264 citrandarin; F.80-18, F.80-3, F.80-5, F.80-6, F.80-7, F.80-8, 'W-2', and 'Swingle' citrumelos $(C$. paradisi $\times P$. trifoliata $)$; 'Rangpur' lime $\times$ sour orange $(C$. limonia $\times$ Citrus aurantium L.); 'US-802' pummelo hybrid (Citrus grandis Hassk. 'Siamese' $\times P$. trifoliata 'Gotha Road'); 'Murcott' tangor $\times$ trifoliate-9 $[(C$. reticulata $\times C$. sinensis $) \times P$. trifoliata $]$; 'Flying Dragon' trifoliate orange (P. trifoliata var. monstrosa); trifoliate orange; and 'Rangpur' lime. IPEACS-256 and IPEACS-264 citrandarins were registered as cultivars Indio and Riverside, respectively, by Empresa Brasileira de Pesquisa Agropecuária (Embrapa) (Rodrigues et al., 2015).

Tree growth development, production, and orange juice chemical and industrial properties were assessed following the procedures described by Domingues et al. (2021b) and Association of Official Analytical Chemists (AOAC) (Latimer, 2019). Tree growth development (height, diameter, and canopy volume) was evaluated in mid-fall, from the 2016 to 2018 harvest seasons. Production per tree was determined from 2016 to 2019, and productive efficiency was obtained from 2016 to 2018. The chemical composition (soluble solids, titratable acidity, and maturation index or ratio) and industrial properties (juice yield, technological index, and industrial index) of the orange juices were evaluated from the 2017 to 2019 harvest seasons by the Integrada Cooperative in the municipality of Uraí, in the state of Paraná, Brazil, according to AOAC (Latimer, 2019).

Data were subjected to the analysis of variance, and means were grouped by the Scott-Knott test, at 5\% probability, using the Sisvar software (Ferreira, 2011). In addition, the principal component analysis (PCA) was performed to explain the interrelationships between the rootstocks regarding the presented variables (Costa et al., 2020), which were subsequently grouped using hierarchical cluster classification (HCC). The R software was used to perform the multivariate analyses (PCA and HCC) with the aid of the FactorMineR package (R Core Team, 2019).

A quick reference chart was proposed based on Castle et al. (1993) and using: cluster analysis of production per tree; productive efficiency; technological index; industrial yield in the 2016, 2017, and 2018 harvest seasons; and canopy volume in the 2018 harvest season.

\section{Results and Discussion}

Regarding tree growth development in 2018, when trees reached their mature stage (Table 1), the canopy height of 'Valência' sweet orange showed the lowest means on F.80-18 citrumelo and 'Flying Dragon' trifoliate orange, but the highest ones on 'Rangpur' lime $\times$ sour orange and 'Rangpur' lime. Regarding canopy diameter, the lowest means of $<2.2 \mathrm{~m}$ were found for trees grafted on 'Flying Dragon' trifoliate orange and on F.80-18, F.80-3, and F.80-5 citrumelos, whereas the highest means were observed on 'US-852', 'US-801', 'US-812', and IPEACS-256 citrandarins, 'Rangpur' lime $\times$ sour orange, and 'Rangpur' lime. Trees on F.80-18 and F.80-5 citrumelos and 'Flying Dragon' trifoliate showed the lowest canopy volumes of $<4.1 \mathrm{~m}^{3}$, while those on 'Rangpur' lime, 'Rangpur' lime $\times$ sour orange, and IPEACS-264 citrandarin were the most vigorous. On average, the canopy volume on these rootstocks was $\approx 200$ and $100 \%$ lower than on the 'Rangpur' lime and 'Swingle' citrumelo commercial rootstocks, respectively.

According to Costa et al. (2020), citrandarin and citrumelo rootstocks, evaluated in several trials and different environments, presented an interesting growth development and were among the most promising new hybrid rootstocks for sweet orange trees. In the present work, some genotypes showed a competitive performance in relation to 'Rangpur' lime under rainfed cultivation in subtropical climate (Cfa type) conditions.

When choosing scion/rootstock combinations, those that tend to take longer for the tree canopies to begin to overlap and form a hedgerow are preferred, because they would reduce the need for mechanical hedge pruning in the first years of tree growth development. Canopy overlap between rows hinders management practices and harvesting operations such as the transit of pickers, placement of harvest boxes for the temporary storage of fruit, and entry of agricultural machinery. 
Therefore, frequent pruning is required to avoid overlap in high-density planting, and scion/rootstock combinations with shorter and narrower canopies may reduce the need for pruning in the early years of orchard establishment. For instance, in the present trial, due to the low canopy volume of 'Valência' sweet orange trees induced by F.80-18 and F.80-5 citrumelos and 'Flying Dragon' trifoliate orange, hedge pruning may only be necessary after the fifth year of orchard planting, considering the used spacing. Therefore, to establish 'Valência' orange orchards with small and low-vigor trees, F.80-18 and F.80-5 citrumelos can be considered alternative rootstocks, with dwarfing characteristics similar to those of 'Flying Dragon' trifoliate orange. It should be noted that, during the assessment period, none of the selected rootstocks presented graft incompatibility with 'Valência' sweet orange.

The highest means for accumulated production per tree along the seasons (Table 2) were obtained on 'US-852', 'US-801', IPEACS-256, and IPEACS-264 citrandarins, as well as on 'Rangpur' lime $\times$ sour orange and 'Rangpur' lime, while the lowest ones were observed on 'Frying Dragon' trifoliate orange. However, in 2018, production efficiency was the highest for trees grafted on F.80-18, followed by those on F.80-3 and F.80-5 citrumelos, with values that were even higher than those on 'Rangpur' lime and 'Swingle' citrumelo (Table 3). Production efficiency was, on average, $\approx 200$ and $300 \%$ higher on F.80-18, F.80-3, and F.80-5 citrumelos, compared with 'Rangpur' lime and 'Swingle' citrumelo, respectively. Under different

Table 1. Tree growth development of 'Valência' sweet orange (Citrus sinensis) grafted on 20 rootstocks in the 2016, 2017, and 2018 harvest seasons ${ }^{(1)}$.

\begin{tabular}{|c|c|c|c|c|c|c|c|c|c|}
\hline \multirow[t]{2}{*}{ Rootstock $^{(2)}$} & \multicolumn{3}{|c|}{ Canopy height (m) } & \multicolumn{3}{|c|}{ Canopy diameter (m) } & \multicolumn{3}{|c|}{ Canopy volume $\left(\mathrm{m}^{3}\right)$} \\
\hline & 2016 & 2017 & 2018 & 2016 & 2017 & 2018 & 2016 & 2017 & 2018 \\
\hline 'US-852' citrandarin & $2.9 \mathrm{a}$ & $3.1 \mathrm{~b}$ & $3.5 \mathrm{c}$ & $2.1 b$ & $2.4 \mathrm{a}$ & $2.5 \mathrm{a}$ & $7.2 \mathrm{~b}$ & $9.3 b$ & $11.6 \mathrm{~b}$ \\
\hline 'US-801' citrandarin & $2.9 \mathrm{a}$ & $3.2 \mathrm{~b}$ & $3.6 \mathrm{c}$ & $2.3 \mathrm{a}$ & $2.4 \mathrm{a}$ & $2.6 \mathrm{a}$ & $8.1 \mathrm{a}$ & $9.6 \mathrm{~b}$ & $11.9 \mathrm{~b}$ \\
\hline 'US-812' citrandarin & $2.7 \mathrm{~b}$ & $2.9 \mathrm{c}$ & $3.0 \mathrm{e}$ & $2.2 \mathrm{a}$ & $2.4 \mathrm{a}$ & $2.5 \mathrm{a}$ & $6.9 \mathrm{~b}$ & $8.5 \mathrm{c}$ & $9.5 \mathrm{c}$ \\
\hline IPEACS-256 citrandarin & $2.9 \mathrm{a}$ & $2.9 \mathrm{c}$ & $3.4 \mathrm{c}$ & $2.3 \mathrm{a}$ & $2.4 \mathrm{a}$ & $2.5 \mathrm{a}$ & $7.7 \mathrm{a}$ & $8.8 \mathrm{c}$ & $11.1 \mathrm{~b}$ \\
\hline IPEACS-239 citrandarin & $2.2 \mathrm{~d}$ & $2.4 \mathrm{~d}$ & $2.7 \mathrm{e}$ & $2.1 \mathrm{~b}$ & $2.3 \mathrm{a}$ & $2.3 b$ & $5.2 \mathrm{c}$ & $6.2 \mathrm{~d}$ & $7.4 \mathrm{~d}$ \\
\hline IPEACS-264 citrandarin & $2.9 \mathrm{a}$ & $3.1 \mathrm{~b}$ & $3.8 \mathrm{~b}$ & $2.2 \mathrm{a}$ & $2.4 \mathrm{a}$ & $2.6 \mathrm{a}$ & $7.5 \mathrm{a}$ & $9.5 b$ & $12.7 \mathrm{a}$ \\
\hline F.80-18 citrumelo & $1.7 \mathrm{e}$ & $1.9 \mathrm{e}$ & $2.1 \mathrm{~g}$ & $1.6 \mathrm{~d}$ & $1.9 \mathrm{c}$ & $2.0 \mathrm{~d}$ & $2.3 \mathrm{e}$ & $3.6 \mathrm{e}$ & $4.1 \mathrm{e}$ \\
\hline F.80-3 citrumelo & $2.2 \mathrm{~d}$ & $2.4 \mathrm{~d}$ & $2.7 \mathrm{e}$ & $1.9 \mathrm{c}$ & $2.2 b$ & $2.2 \mathrm{c}$ & $4.4 \mathrm{c}$ & $5.9 \mathrm{~d}$ & $6.6 \mathrm{~d}$ \\
\hline F.80-5 citrumelo & $2.0 \mathrm{~d}$ & $2.4 \mathrm{~d}$ & $2.4 \mathrm{f}$ & $1.9 \mathrm{c}$ & $2.1 \mathrm{~b}$ & $2.1 \mathrm{c}$ & $3.8 \mathrm{~d}$ & $5.1 d$ & $5.4 \mathrm{e}$ \\
\hline F.80-6 citrumelo & $2.5 b$ & $2.7 \mathrm{c}$ & $3.1 \mathrm{~d}$ & $2.1 \mathrm{~b}$ & $2.4 \mathrm{a}$ & $2.4 \mathrm{~b}$ & $5.8 b$ & $8.2 \mathrm{c}$ & $9.5 \mathrm{c}$ \\
\hline F.80-7 citrumelo & $2.8 \mathrm{~b}$ & $3.3 b$ & $3.8 \mathrm{~b}$ & $2.1 \mathrm{~b}$ & $2.4 \mathrm{a}$ & $2.4 \mathrm{~b}$ & $6.7 b$ & $9.3 b$ & $11.8 \mathrm{~b}$ \\
\hline F.80-8 citrumelo & $2.4 \mathrm{c}$ & $2.8 \mathrm{c}$ & $3.2 \mathrm{~d}$ & $2.1 \mathrm{~b}$ & $2.3 \mathrm{a}$ & $2.4 \mathrm{~b}$ & $5.5 \mathrm{c}$ & $7.7 \mathrm{c}$ & $9.5 \mathrm{c}$ \\
\hline 'W-2' citrumelo & $2.7 \mathrm{~b}$ & $3.0 \mathrm{c}$ & $3.7 \mathrm{~b}$ & $2.1 \mathrm{~b}$ & $2.4 \mathrm{a}$ & $2.5 \mathrm{a}$ & $6.7 b$ & $9.1 b$ & $12.1 \mathrm{~b}$ \\
\hline 'Swingle' citrumelo & $2.6 \mathrm{~b}$ & $2.9 \mathrm{c}$ & $3.5 \mathrm{c}$ & $2.1 \mathrm{~b}$ & $2.4 \mathrm{a}$ & $2.5 \mathrm{a}$ & $6.2 b$ & $8.7 \mathrm{c}$ & $11.2 \mathrm{~b}$ \\
\hline 'Rangpur' lime $\times$ sour orange & $3.1 \mathrm{a}$ & $3.5 \mathrm{a}$ & $4.2 \mathrm{a}$ & $2.3 \mathrm{a}$ & $2.5 \mathrm{a}$ & $2.6 \mathrm{a}$ & $8.8 \mathrm{a}$ & $10.8 \mathrm{a}$ & $14.6 \mathrm{a}$ \\
\hline 'US-802' pummelo hybrid & $2.6 \mathrm{~b}$ & $2.7 \mathrm{c}$ & $3.0 \mathrm{e}$ & $2.0 \mathrm{c}$ & $2.4 \mathrm{a}$ & $2.4 \mathrm{~b}$ & $5.4 \mathrm{c}$ & $7.9 \mathrm{c}$ & $8.9 \mathrm{c}$ \\
\hline 'Murcott' tangor $\times$ trifoliate- 9 & $2.6 \mathrm{~b}$ & $2.8 \mathrm{c}$ & $3.2 \mathrm{~d}$ & $2.1 \mathrm{~b}$ & $2.3 \mathrm{a}$ & $2.4 \mathrm{~b}$ & $5.9 \mathrm{~b}$ & $7.5 \mathrm{c}$ & $9.0 \mathrm{c}$ \\
\hline Trifoliate orange & $2.4 \mathrm{c}$ & $2.5 \mathrm{~d}$ & $2.7 \mathrm{e}$ & $2.0 \mathrm{c}$ & $2.4 \mathrm{a}$ & $2.4 \mathrm{~b}$ & $4.8 \mathrm{c}$ & $6.8 \mathrm{~d}$ & $8.0 \mathrm{~d}$ \\
\hline 'Flying Dragon' trifoliate orange & $1.7 \mathrm{e}$ & $1.9 \mathrm{e}$ & $2.0 \mathrm{~g}$ & $1.7 \mathrm{~d}$ & $1.9 \mathrm{c}$ & $1.9 \mathrm{~d}$ & $2.5 \mathrm{e}$ & $3.4 \mathrm{e}$ & $3.7 \mathrm{e}$ \\
\hline 'Rangpur' lime & $3.1 \mathrm{a}$ & $3.5 \mathrm{a}$ & $3.9 \mathrm{~b}$ & $2.3 \mathrm{a}$ & $2.5 \mathrm{a}$ & $2.6 \mathrm{a}$ & $8.3 \mathrm{a}$ & $10.9 \mathrm{a}$ & $13.4 \mathrm{a}$ \\
\hline F-value & $25.3 * *$ & $23.8 * *$ & $24.0 * *$ & $9.7 * *$ & $12.6^{* *}$ & $14.5 * *$ & $19.2 * *$ & $22.6 * *$ & $23.3 * *$ \\
\hline
\end{tabular}

${ }^{(1)}$ Means followed by equal letters do not differ by the Scott-Knott test, at $5 \%$ probability. Trees were planted in April, 2013. ${ }^{(2)}$ Species: citrandarin, Citrus reticulata $\times$ Poncirus trifoliata; citrumelo, Citrus paradisi $\times$ P. trifoliata; 'Rangpur' lime $\times$ sour orange, Citrus limonia $\times$ Citrus aurantium; 'US-802' pummelo hybrid, Citrus grandis $\times$ P. trifoliata; 'Murcott' tangor $\times$ trifoliate-9, C. reticulata $\times$ C. sinensis; trifoliate orange, $P$. trifoliata; 'Flying Dragon' trifoliate orange, P. trifoliata var. monstrosa; and 'Rangpur' lime, C. limonia. CV, coefficient of variation. **Significant at $1 \%$ probability. 
environmental conditions, those rootstocks also induced a higher production efficiency for 'Valência' sweet orange than 'Rangpur' lime and 'Swingle' citrumelo, but in a lower proportion (Pompeu Júnior $\&$ Blumer, 2011). Therefore, the evaluation of the performance of rootstocks in different edaphoclimatic conditions is essential to identify those that gather the most desirable features for each site.

Under the environmental conditions of this trial, high-density plantings with rootstocks with a low vigor and high production efficiency, such as F.80-18, F.80-5, and F.80-3 citrumelos, could make the production system of new 'Valência' sweet orange orchards more feasible, with greater productivity earnings. This is important since high-density planting is one of the strategies used to mitigate HLB - the main disease that currently affects orange orchards worldwide -, allowing the removal of symptomatic trees (Vashisth et al., 2020).

In the last season assessed, when 'Valência' orange trees were considered mature, the highest means for the orange juice maturation index or ratio, which is directly influenced by the soluble solids and titratable acidity contents of the orange juices, were observed on IPEACS-256 citrandarin, F80-18, F.80-3, F.805, F.80-6, F.80-8, and 'Swingle' citrumelos, 'US802' pummelo hybrid, and 'Flying Dragon' trifoliate orange; the obtained values were higher than those on 'Rangpur' lime (Table 4). In addition, the rootstocks had no influence on juice yield, but the industrial yield

Table 2. Production per tree and accumulated production per tree of 'Valência' sweet orange (Citrus sinensis) grafted on 20 rootstocks in the 2016, 2017, 2018, and 2019 harvest seasons ${ }^{(1)}$.

\begin{tabular}{|c|c|c|c|c|c|}
\hline \multirow[t]{2}{*}{ Rootstock $^{(2)}$} & \multicolumn{4}{|c|}{ Production per tree (kg per tree) } & \multirow{2}{*}{$\begin{array}{l}\text { Accumulated production } \\
\text { (kg per tree) }\end{array}$} \\
\hline & 2016 & 2017 & 2018 & 2019 & \\
\hline 'US-852' citrandarin & $36.5 \mathrm{~d}$ & $115.8 \mathrm{a}$ & $123.8 \mathrm{a}$ & $72.2 \mathrm{a}$ & $348.3 \mathrm{a}$ \\
\hline 'US-801' citrandarin & $47.9 \mathrm{~d}$ & $134.9 \mathrm{a}$ & $104.0 \mathrm{a}$ & $88.5 \mathrm{a}$ & $375.3 \mathrm{a}$ \\
\hline 'US-812' citrandarin & $58.6 \mathrm{c}$ & $111.0 \mathrm{a}$ & $84.3 b$ & $55.5 \mathrm{~b}$ & $309.3 b$ \\
\hline IPEACS-256 citrandarin & $71.6 \mathrm{~b}$ & $122.6 \mathrm{a}$ & $111.2 \mathrm{a}$ & $58.5 \mathrm{~b}$ & $363.9 \mathrm{a}$ \\
\hline IPEACS-239 citrandarin & $62.7 \mathrm{c}$ & $79.5 b$ & $69.5 \mathrm{c}$ & $54.6 \mathrm{~b}$ & $266.3 \mathrm{c}$ \\
\hline IPEACS-264 citrandarin & $61.4 \mathrm{c}$ & $123.0 \mathrm{a}$ & $104.0 \mathrm{a}$ & $70.6 \mathrm{a}$ & $359.1 \mathrm{a}$ \\
\hline F.80-18 citrumelo & $23.5 \mathrm{e}$ & $40.1 \mathrm{c}$ & $107.0 \mathrm{a}$ & $42.5 b$ & $213.0 \mathrm{~d}$ \\
\hline F.80-3 citrumelo & $47.7 \mathrm{~d}$ & $77.7 \mathrm{~b}$ & $103.6 \mathrm{a}$ & $72.2 \mathrm{a}$ & $296.2 b$ \\
\hline F.80-5 citrumelo & $42.0 \mathrm{~d}$ & $60.8 \mathrm{~b}$ & $89.5 b$ & $50.5 b$ & $242.8 \mathrm{c}$ \\
\hline F.80-6 citrumelo & $33.3 \mathrm{e}$ & $80.3 b$ & $82.5 b$ & $77.6 \mathrm{a}$ & $273.6 \mathrm{c}$ \\
\hline F.80-7 citrumelo & $53.7 \mathrm{c}$ & $113.9 \mathrm{a}$ & $53.7 \mathrm{~d}$ & $77.9 \mathrm{a}$ & $299.0 \mathrm{~b}$ \\
\hline F.80-8 citrumelo & $41.2 \mathrm{~d}$ & $78.0 \mathrm{~b}$ & $40.4 \mathrm{e}$ & $68.5 \mathrm{a}$ & $227.3 \mathrm{c}$ \\
\hline 'W-2' citrumelo & $57.1 \mathrm{c}$ & $119.5 \mathrm{a}$ & $57.1 \mathrm{~d}$ & $94.1 \mathrm{a}$ & $327.8 b$ \\
\hline 'Swingle' citrumelo & $47.7 \mathrm{~d}$ & $105.8 \mathrm{a}$ & $47.7 \mathrm{~d}$ & $84.1 \mathrm{a}$ & $285.3 b$ \\
\hline 'Rangpur' lime $\times$ sour orange & $68.2 b$ & $134.5 \mathrm{a}$ & $68.2 \mathrm{c}$ & $86.9 \mathrm{a}$ & $357.7 \mathrm{a}$ \\
\hline 'US-802' pummelo hybrid & $25.9 \mathrm{e}$ & $73.4 \mathrm{~b}$ & $25.9 \mathrm{e}$ & $71.9 \mathrm{a}$ & $198.0 \mathrm{~d}$ \\
\hline 'Murcott' tangor $\times$ trifoliate- 9 & $51.4 \mathrm{c}$ & $102.6 \mathrm{a}$ & $51.4 \mathrm{~d}$ & $58.0 \mathrm{~b}$ & $263.4 \mathrm{c}$ \\
\hline Trifoliate orange & $31.0 \mathrm{e}$ & $77.0 \mathrm{~b}$ & $30.9 \mathrm{e}$ & $64.2 \mathrm{~b}$ & $203.0 \mathrm{~d}$ \\
\hline 'Flying Dragon' trifoliate orange & $36.4 \mathrm{~d}$ & $35.7 \mathrm{c}$ & $36.4 \mathrm{e}$ & $25.5 b$ & $134.0 \mathrm{e}$ \\
\hline 'Rangpur' lime & $83.6 \mathrm{a}$ & $144.1 \mathrm{a}$ & $83.6 \mathrm{~b}$ & $105.9 \mathrm{a}$ & $417.1 \mathrm{a}$ \\
\hline F-value & $14.3^{* *}$ & $11.8^{* *}$ & $23.5^{* *}$ & $3.4 * *$ & $11.5^{* *}$ \\
\hline CV (\%) & 17.3 & 18.8 & 16.6 & 29.4 & 14.7 \\
\hline
\end{tabular}

${ }^{(1)}$ Means followed by equal letters do not differ by the Scott-Knott test, at $5 \%$ probability. Trees were planted in April, 2013. ${ }^{(2)}$ Species: citrandarin, Citrus reticulata $\times$ Poncirus trifoliata; citrumelo, Citrus paradisi $\times$ P. trifoliata; 'Rangpur' lime $\times$ sour orange, Citrus limonia $\times$ Citrus aurantium; 'US-802' pummelo hybrid, Citrus grandis $\times$ P. trifoliata; 'Murcott' tangor $\times$ trifoliate-9, C. reticulata $\times$ C. sinensis; trifoliate orange, $P$. trifoliata; 'Flying Dragon' trifoliate orange, $P$. trifoliata var. monstrosa; and 'Rangpur' lime, C. limonia. CV, coefficient of variation. **Significant at $1 \%$ probability. 
and the technological index of orange juices were affected, with the best performance being verified for trees grafted on 'US-801', 'US-812', IPEACS-256, IPEACS-239, and IPEACS-264 citrandarins, F.80-5, F.80-8, and 'Swingle' citrumelos, 'US-802' pummelo hybrid, trifoliate orange, and 'Flying Dragon' trifoliate orange, whose values were superior than those on 'Rangpur' lime and 'US-852' citrandarin (Table 4). In other words, when 'Valência' sweet orange trees were grafted on those rootstock genotypes, compared with on 'Rangpur' lime, there was a lower demand $(\approx 10 \%$ less) for orange boxes to produce a ton of frozen concentrated orange juice, implicating a better processing efficiency.

Table 3. Productive efficiency of 'Valência' sweet orange (Citrus sinensis) trees grafted on 20 rootstocks in the 2016, 2017, and 2018 harvest seasons ${ }^{(1)}$.

\begin{tabular}{lccc}
\hline Rootstock & \multicolumn{3}{c}{ Productive efficiency $\left(\mathrm{kg} \mathrm{m}^{-3}\right)$} \\
\cline { 2 - 4 } & 2016 & 2017 & 2018 \\
\hline 'US-852' citrandarin & $5.1 \mathrm{~d}$ & $12.6 \mathrm{a}$ & $10.6 \mathrm{c}$ \\
'US-801' citrandarin & $6.0 \mathrm{~d}$ & $14.1 \mathrm{a}$ & $9.0 \mathrm{c}$ \\
'US-812' citrandarin & $8.8 \mathrm{c}$ & $13.0 \mathrm{a}$ & $8.7 \mathrm{c}$ \\
IPEACS-256 citrandarin & $9.4 \mathrm{c}$ & $13.9 \mathrm{a}$ & $10.0 \mathrm{c}$ \\
IPEACS-239 citrandarin & $13.4 \mathrm{a}$ & $13.0 \mathrm{a}$ & $9.6 \mathrm{c}$ \\
IPEACS264 citrandarin & $8.3 \mathrm{c}$ & $12.8 \mathrm{a}$ & $8.1 \mathrm{c}$ \\
F.80-18 citrumelo & $10.6 \mathrm{~b}$ & $11.1 \mathrm{~b}$ & $26.6 \mathrm{a}$ \\
F.80-3 citrumelo & $9.9 \mathrm{~b}$ & $13.7 \mathrm{a}$ & $15.9 \mathrm{~b}$ \\
F.80-5 citrumelo & $11.9 \mathrm{~b}$ & $12.1 \mathrm{a}$ & $16.8 \mathrm{~b}$ \\
F.80-6 citrumelo & $5.5 \mathrm{~d}$ & $9.7 \mathrm{~b}$ & $8.8 \mathrm{c}$ \\
F.80-7 citrumelo & $8.5 \mathrm{c}$ & $12.2 \mathrm{a}$ & $4.7 \mathrm{~d}$ \\
F.80-8 citrumelo & $7.7 \mathrm{c}$ & $10.4 \mathrm{~b}$ & $4.4 \mathrm{~d}$ \\
'W-2' citrumelo & $8.7 \mathrm{c}$ & $13.1 \mathrm{a}$ & $4.7 \mathrm{~d}$ \\
'Swingle' citrumelo & $8.1 \mathrm{c}$ & $12.3 \mathrm{a}$ & $4.4 \mathrm{~d}$ \\
'Rangpur' lime $\times$ sour orange & $7.8 \mathrm{c}$ & $12.5 \mathrm{a}$ & $4.7 \mathrm{~d}$ \\
'US-802' pummelo hybrid & $5.2 \mathrm{~d}$ & $9.3 \mathrm{~b}$ & $2.9 \mathrm{~d}$ \\
'Murcott' tangor $\times$ trifoliate-9 & $9.1 \mathrm{c}$ & $13.7 \mathrm{a}$ & $5.7 \mathrm{~d}$ \\
Trifoliate orange & $6.6 \mathrm{~d}$ & $11.4 \mathrm{~b}$ & $3.9 \mathrm{~d}$ \\
'Flying Dragon' trifoliate orange & $14.9 \mathrm{a}$ & $10.5 \mathrm{~b}$ & $9.9 \mathrm{c}$ \\
'Rangpur' lime & $10.1 \mathrm{~b}$ & $13.2 \mathrm{a}$ & $6.2 \mathrm{~d}$ \\
\hline F-value & $6.0 * *$ & $1.7 *$ & $36.2 * *$ \\
CV $(\%)$ & 24.1 & 17.6 & 21.4 \\
\hline
\end{tabular}

${ }^{(1)}$ Means followed by equal letters do not differ by the Scott-Knott test, at $5 \%$ probability. Trees were planted in April, 2013. ${ }^{(2)}$ Species: citrandarin, Citrus reticulata $\times$ Poncirus trifoliata; citrumelo, Citrus paradisi $\times$ P. trifoliata; 'Rangpur' lime $\times$ sour orange, Citrus limonia $\times$ Citrus aurantium; 'US-802' pummelo hybrid, Citrus grandis $\times$ P. trifoliata; 'Murcott' tangor $\times$ trifoliate-9, C. reticulata $\times$ C. sinensis; trifoliate orange, P. trifoliata; 'Flying Dragon' trifoliate orange, P. trifoliata var. monstrosa; and 'Rangpur' lime, C. limonia. CV, coefficient of variation. $*$ and ${ }^{*}$ Significant at 5 and $1 \%$ probability, respectively.
For the principal component analysis, the industrial yield and technological index had greater contributions, showing a negative correlation between both of them (Figure 1). Yield (production per tree) was positively correlated with canopy volume, indicating that vigorous trees had higher yields, but was negatively correlated with productive efficiency, as also reported by Fadel et al. (2018) and Rodrigues et al. (2019).

It was possible to identify four groups of rootstocks. The first two groups induced better quality 'Valência' orange fruits and were composed of: 'US-801', 'US812', IPEACS-239, IPEACS-256, and IPEACS-264 citrandarins; and F.80-3, F.80-5, F.80-6, F.80-8, and 'Swingle' citrumelos, 'US-802' pummelo hybrid, trifoliate orange, and 'Flying Dragon' trifoliate orange. The third group was composed of 'US-852' citrandarin, F.807 and 'W2' citrumelos, 'Rangpur' lime, 'Murcott' tangor $\times$ trifoliate-9, and 'Rangpur' lime $\times$ sour orange, which induced a higher production, but were vigorous and had an inferior performance regarding fruit quality. The last group was composed of F.8018 citrumelo, completely isolated from the other rootstock genotypes, showing a better production efficiency. The multivariate analysis provided more comprehensive information about the performance of the assessed rootstocks, identifying homogenous groups of genotypes, considering the universe of all characteristics that were significant by the univariate analysis of variance, as also described by Costa et al. (2020, 2021).

Based on the findings of the present study, a quick reference chart was proposed, accounting for the influence of each rootstock on the main characteristics of 'Valência' sweet orange trees, aiming for a practical and objective identification of the best rootstock alternatives (Table 5). In particular, for high-density plantings, F.80-5 citrumelo is an interesting option because it induces dwarf trees, a high productive efficiency, and a high industrial quality of fruits, followed by F.80-3 citrumelo and IPEACS-239 citrandarin. Moreover, in scenarios where the establishment of low-density plantings is preferred, 'US-801', 'US-812', and IPEACS-256 citrandarins are better options than 'Rangpur' lime and 'Swingle' citrumelo because 'Valência' sweet orange trees grafted on those genotypes have a lower tree size and a better yield and industrial quality. 
Table 4. Chemical and industrial properties of juices of 'Valência' sweet orange (Citrus sinensis) trees grafted on 20 rootstocks in the 2017, 2018, and 2019 harvest seasons ${ }^{(1)}$.

\begin{tabular}{|c|c|c|c|c|c|c|c|c|c|}
\hline \multirow[t]{2}{*}{ Rootstock $^{(2)}$} & \multicolumn{3}{|c|}{ Soluble solids - SS ( ${ }^{\circ}$ Brix) } & \multicolumn{3}{|c|}{ Titratable acidity - TA (\%) } & \multicolumn{3}{|c|}{ Maturation index (SS/TA) } \\
\hline & 2017 & 2018 & 2019 & 2017 & 2018 & 2019 & 2017 & 2018 & 2019 \\
\hline 'US-852' citrandarin & $9.9 \mathrm{~b}$ & 9.1 & $12.1 \mathrm{~b}$ & 0.9 & $0.5 b$ & $0.8 \mathrm{a}$ & $10.9 \mathrm{~b}$ & 18.6 & $15.3 \mathrm{~b}$ \\
\hline 'US-801' citrandarin & $9.8 \mathrm{~b}$ & 9.6 & $11.6 \mathrm{~b}$ & 0.9 & $0.5 b$ & $0.7 b$ & $11.2 \mathrm{~b}$ & 19.6 & $16.0 \mathrm{~b}$ \\
\hline 'US-812' citrandarin & $10.6 \mathrm{a}$ & 9.9 & $12.4 \mathrm{a}$ & 0.9 & $0.6 \mathrm{a}$ & $0.8 \mathrm{a}$ & $11.6 \mathrm{~b}$ & 18.7 & $15.4 \mathrm{~b}$ \\
\hline IPEACS-256 citrandarin & $10.4 \mathrm{a}$ & 9.7 & $12.7 \mathrm{a}$ & 0.9 & $0.5 \mathrm{~b}$ & $0.8 \mathrm{a}$ & $11.7 \mathrm{~b}$ & 20.0 & $16.6 \mathrm{a}$ \\
\hline IPEACS-239 citrandarin & $10.7 \mathrm{a}$ & 9.9 & $12.7 \mathrm{a}$ & 1.0 & $0.6 \mathrm{a}$ & $0.8 \mathrm{a}$ & $10.8 \mathrm{~b}$ & 16.8 & $15.6 \mathrm{~b}$ \\
\hline IPEACS-264 citrandarin & $10.4 \mathrm{a}$ & 9.5 & $12.5 \mathrm{a}$ & 0.9 & $0.5 b$ & $0.9 \mathrm{a}$ & $11.2 \mathrm{~b}$ & 18.1 & $14.0 \mathrm{~b}$ \\
\hline F.80-18 citrumelo & $10.5 \mathrm{a}$ & 8.8 & $12.1 \mathrm{~b}$ & 1.0 & $0.5 b$ & $0.7 b$ & $10.8 \mathrm{~b}$ & 16.9 & $16.2 \mathrm{a}$ \\
\hline F.80-3 citrumelo & $10.7 \mathrm{a}$ & 9.4 & $11.4 \mathrm{~b}$ & 0.9 & $0.5 b$ & $0.7 b$ & $12.2 \mathrm{a}$ & 18.5 & $16.5 \mathrm{a}$ \\
\hline F.80-5 citrumelo & $10.6 \mathrm{a}$ & 9.6 & $11.6 \mathrm{~b}$ & 0.8 & $0.5 b$ & $0.7 \mathrm{~b}$ & $13.0 \mathrm{a}$ & 20.7 & $17.4 \mathrm{a}$ \\
\hline F.80-6 citrumelo & $10.8 \mathrm{a}$ & 9.3 & $12.6 \mathrm{a}$ & 0.8 & $0.5 b$ & $0.7 \mathrm{~b}$ & $13.6 \mathrm{a}$ & 19.1 & $19.5 \mathrm{a}$ \\
\hline F.80-7 citrumelo & $10.2 \mathrm{a}$ & 8.8 & $12.5 \mathrm{a}$ & 0.9 & $0.5 b$ & $0.8 \mathrm{a}$ & $11.2 \mathrm{~b}$ & 17.3 & $15.0 \mathrm{~b}$ \\
\hline F.80-8 citrumelo & $10.7 \mathrm{a}$ & 9.8 & $12.8 \mathrm{a}$ & 0.8 & $0.5 b$ & $0.7 \mathrm{~b}$ & $12.9 \mathrm{a}$ & 20.5 & $17.9 \mathrm{a}$ \\
\hline 'W-2' citrumelo & $10.4 \mathrm{a}$ & 9.3 & $12.3 \mathrm{a}$ & 0.9 & $0.5 b$ & $0.8 \mathrm{a}$ & $12.2 \mathrm{a}$ & 18.3 & $15.1 \mathrm{~b}$ \\
\hline 'Swingle' citrumelo & $10.9 \mathrm{a}$ & 9.5 & $13.3 \mathrm{a}$ & 0.8 & $0.5 b$ & $0.8 \mathrm{a}$ & $13.0 \mathrm{a}$ & 20.2 & $17.1 \mathrm{a}$ \\
\hline 'Rangpur' lime $\times$ sour orange & $9.5 b$ & 8.9 & $11.8 \mathrm{~b}$ & 0.9 & $0.5 b$ & $0.9 \mathrm{a}$ & $11.1 \mathrm{~b}$ & 18.4 & $13.6 \mathrm{~b}$ \\
\hline 'US-802' pummelo hybrid & $10.8 \mathrm{a}$ & 9.5 & $12.4 \mathrm{a}$ & 1.0 & $0.5 b$ & $0.7 \mathrm{~b}$ & $11.3 \mathrm{~b}$ & 19.0 & $16.9 \mathrm{a}$ \\
\hline 'Murcott' tangor $\times$ trifoliate- 9 & $9.8 b$ & 9.1 & $12.0 \mathrm{~b}$ & 0.8 & $0.6 \mathrm{a}$ & $0.8 \mathrm{a}$ & $12.0 \mathrm{a}$ & 17.4 & $14.4 \mathrm{~b}$ \\
\hline Trifoliate orange & $10.7 \mathrm{a}$ & 9.3 & $13.2 \mathrm{a}$ & 0.9 & $0.5 b$ & $0.8 \mathrm{a}$ & $11.8 \mathrm{a}$ & 18.1 & $15.9 \mathrm{~b}$ \\
\hline 'Flying Dragon' trifoliate orange & $11.1 \mathrm{a}$ & 10.4 & $12.7 \mathrm{a}$ & 0.9 & $0.6 \mathrm{a}$ & $0.7 b$ & $12.6 \mathrm{a}$ & 18.7 & $17.6 \mathrm{a}$ \\
\hline 'Rangpur' lime & $9.1 \mathrm{~b}$ & 8.8 & $11.4 \mathrm{~b}$ & 0.8 & $0.5 \mathrm{~b}$ & $0.9 \mathrm{a}$ & $11.2 \mathrm{~b}$ & 17.2 & $13.4 \mathrm{~b}$ \\
\hline F-value & $3.9^{*}$ & $2.0^{\mathrm{ns}}$ & $6.0^{*}$ & $1.4^{\mathrm{ns}}$ & $2.0 * *$ & $3.0^{*}$ & $2.3^{*}$ & $1.7^{\mathrm{ns}}$ & $4.0^{*}$ \\
\hline \multirow[t]{2}{*}{ Rootstock $^{(2)}$} & \multicolumn{3}{|c|}{ Juice yield (\%) } & \multicolumn{3}{|c|}{ Industrial yield $^{(3)}$} & \multicolumn{3}{|c|}{ Technological index ${ }^{(4)}$} \\
\hline & 2017 & 2018 & 2019 & 2017 & 2018 & 2019 & 2017 & 2018 & 2019 \\
\hline 'US-852' citrandarin & 63.2 & $50.7 \mathrm{a}$ & 55.5 & 259.3 & $353.6 \mathrm{a}$ & $242.0 \mathrm{a}$ & 2.6 & $1.9 \mathrm{~b}$ & $2.7 \mathrm{~b}$ \\
\hline 'US-801' citrandarin & 64.5 & $52.9 \mathrm{a}$ & 61.5 & 259.3 & $317.0 \mathrm{~b}$ & $226.1 \mathrm{~b}$ & 2.6 & $2.1 \mathrm{a}$ & $2.9 \mathrm{a}$ \\
\hline 'US-812' citrandarin & 58.0 & $55.1 \mathrm{a}$ & 57.3 & 266.8 & $300.3 b$ & $230.2 b$ & 2.5 & $2.2 \mathrm{a}$ & $2.9 \mathrm{a}$ \\
\hline IPEACS-256 citrandarin & 56.6 & $56.0 \mathrm{a}$ & 60.5 & 279.4 & $299.0 \mathrm{~b}$ & $210.9 b$ & 2.4 & $2.2 \mathrm{a}$ & $3.1 \mathrm{a}$ \\
\hline IPEACS-239 citrandarin & 59.9 & $55.1 \mathrm{a}$ & 59.5 & 256.5 & $295.9 b$ & $214.5 b$ & 2.6 & $2.2 \mathrm{a}$ & $3.1 \mathrm{a}$ \\
\hline IPEACS-264 citrandarin & 59.5 & $54.5 \mathrm{a}$ & 58.3 & 262.8 & $314.4 \mathrm{~b}$ & $223.0 \mathrm{~b}$ & 2.5 & $2.1 \mathrm{a}$ & $3.0 \mathrm{a}$ \\
\hline F.80-18 citrumelo & 53.3 & $41.0 \mathrm{c}$ & 54.5 & 295.5 & $410.5 \mathrm{a}$ & $247.8 \mathrm{a}$ & 2.3 & $1.5 \mathrm{~b}$ & $2.7 \mathrm{~b}$ \\
\hline F.80-3 citrumelo & 53.8 & $51.4 \mathrm{a}$ & 54.8 & 283.2 & $337.1 \mathrm{~b}$ & $259.4 \mathrm{a}$ & 2.3 & $2.0 \mathrm{a}$ & $2.6 \mathrm{~b}$ \\
\hline F.80-5 citrumelo & 59.1 & $52.4 \mathrm{a}$ & 61.8 & 260.0 & $326.2 b$ & $226.3 b$ & 2.5 & $2.0 \mathrm{a}$ & $2.9 \mathrm{a}$ \\
\hline F.80-6 citrumelo & 58.4 & $52.0 \mathrm{a}$ & 52.9 & 256.4 & $355.6 \mathrm{a}$ & $244.5 \mathrm{a}$ & 2.6 & $2.0 \mathrm{a}$ & $2.7 \mathrm{~b}$ \\
\hline F.80-7 citrumelo & 55.5 & $52.5 \mathrm{a}$ & 55.4 & 287.9 & $361.0 \mathrm{a}$ & $235.0 \mathrm{a}$ & 2.3 & $1.9 \mathrm{~b}$ & $2.8 \mathrm{~b}$ \\
\hline F.80-8 citrumelo & 57.5 & $54.1 \mathrm{a}$ & 56.4 & 264.4 & $306.7 \mathrm{~b}$ & $224.4 \mathrm{~b}$ & 2.5 & $2.2 \mathrm{a}$ & $2.9 \mathrm{a}$ \\
\hline 'W-2' citrumelo & 54.5 & $53.4 \mathrm{a}$ & 56.1 & 288.9 & $321.1 \mathrm{~b}$ & $236.5 \mathrm{a}$ & 2.3 & $2.0 \mathrm{a}$ & $2.8 \mathrm{~b}$ \\
\hline 'Swingle' citrumelo & 56.1 & $52.4 \mathrm{a}$ & 57.8 & 265.8 & $328.1 \mathrm{~b}$ & $211.6 b$ & 2.5 & $2.0 \mathrm{a}$ & $3.1 \mathrm{a}$ \\
\hline 'Rangpur' lime $\times$ sour orange & 62.6 & $48.7 \mathrm{~b}$ & 57.0 & 272.8 & $375.9 \mathrm{a}$ & $240.0 \mathrm{a}$ & 2.4 & $1.8 \mathrm{~b}$ & $2.8 \mathrm{~b}$ \\
\hline 'US-802' pummelo hybrid & 55.6 & $53.8 \mathrm{a}$ & 58.7 & 272.7 & $316.1 \mathrm{~b}$ & $222.7 \mathrm{~b}$ & 2.4 & $2.1 \mathrm{a}$ & $3.0 \mathrm{a}$ \\
\hline 'Murcott' tangor $\times$ trifoliate- 9 & 60.5 & $44.8 \mathrm{c}$ & 55.3 & 275.4 & $371.6 \mathrm{a}$ & $243.1 \mathrm{a}$ & 2.4 & $1.7 \mathrm{~b}$ & $2.7 \mathrm{~b}$ \\
\hline Trifoliate orange & 58.2 & $47.7 \mathrm{~b}$ & 56.8 & 263.1 & $366.6 \mathrm{a}$ & $216.1 \mathrm{~b}$ & 2.5 & $1.8 \mathrm{~b}$ & $3.0 \mathrm{a}$ \\
\hline 'Flying Dragon' trifoliate orange & 57.0 & $51.0 \mathrm{a}$ & 56.4 & 257.5 & $305.6 \mathrm{~b}$ & $226.7 b$ & 2.6 & $2.2 \mathrm{a}$ & $2.9 \mathrm{a}$ \\
\hline 'Rangpur' lime & 60.8 & $49.5 \mathrm{a}$ & 57.4 & 299.0 & $373.4 \mathrm{a}$ & $249.0 \mathrm{a}$ & 2.3 & $1.8 \mathrm{~b}$ & $2.7 \mathrm{~b}$ \\
\hline F-value & $1.2^{\mathrm{ns}}$ & $3.6^{*}$ & $1.5^{\mathrm{ns}}$ & $0.8^{\mathrm{ns}}$ & $3.2 *$ & $2.4^{*}$ & $0.7^{\text {ns }}$ & $4.3^{*}$ & $2.4^{*}$ \\
\hline
\end{tabular}

(1)Means followed by equal letters do not differ by the Scott-Knott test, at 5\% probability. Trees were planted in April, 2013. ${ }^{(2)}$ Species: citrandarin, Citrus reticulata $\times$ Poncirus trifoliata; citrumelo, Citrus paradisi $\times$ P. trifoliata; 'Rangpur' lime $\times$ sour orange, Citrus limonia $\times$ Citrus aurantium; 'US- 802 ' pummelo hybrid, Citrus grandis $\times$ P. trifoliata; 'Murcott' tangor $\times$ trifoliate-9, C. reticulata $\times$ C. sinensis; trifoliate orange, $P$. trifoliata; 'Flying Dragon' trifoliate orange, P. trifoliata var. monstrosa; and 'Rangpur' lime, C. limonia. CV, coefficient of variation. ${ }^{(3)}$ Number of boxes of $40.8 \mathrm{~kg}$ per ton of frozen concentrated orange juice. ${ }^{(4)}$ Kilogram of SS per box of $40.8 \mathrm{~kg}$. CV, coefficient of variation. * and **Significant at 5 and $1 \%$ probability, respectively. ${ }^{\mathrm{n}}$ Nonsignificant. 

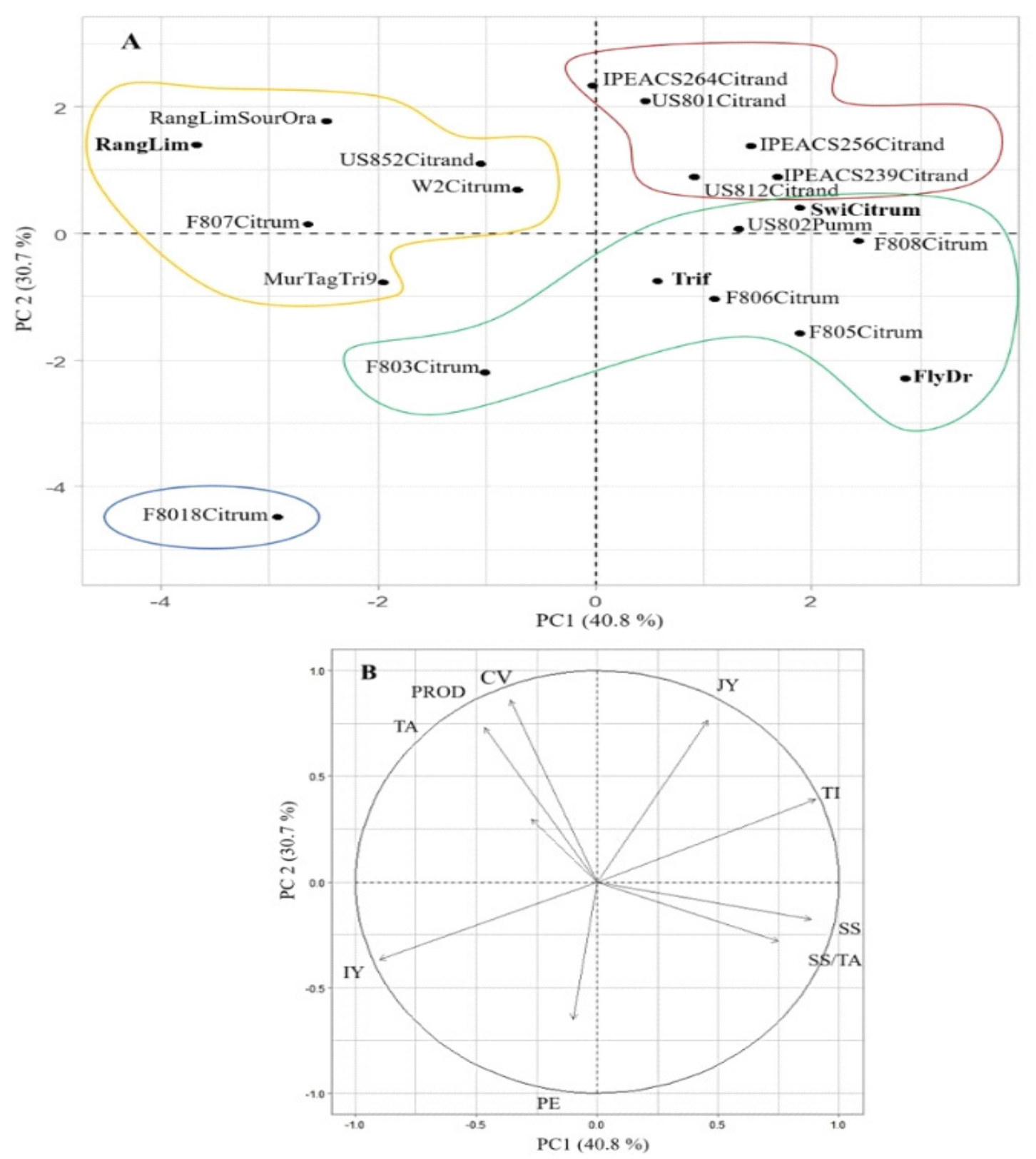

Figure 1. Principal component analysis of production (PROD), canopy volume (CV), production efficiency (PE), technological index (TI), soluble solids (SS), titratable acidity (TA), maturation index or SS/TA ratio, industrial yield (IY), and juice yield (JY) of 'Valência' sweet orange (Citrus sinensis) grafted onto 20 rootstocks, showing: A, treatment dispersion according to the scores of the main components; and B, variable arrangement according to the scores of the main components. US852Citrand, 'US-852' citrandarin; US801Citrand, 'US-801' citrandarin; US812Citrand, 'US-812' citrandarin; IPEACS256Citrand, IPEACS-256 citrandarin; IPEACS239Citrand, IPEACS-239 citrandarin; IPEACS264 Citrand, IPEACS-264 citrandarin; F8018Citrum, F.80-18 citrumelo; F803Citrum, F.80-3 citrumelo; F805Citrum, F.80-5 citrumelo; F806Citrum, F.80-6 citrumelo; F807Citrum, F.80-7 citrumelo; F80-8Citrum, F.80-8 citrumelo; W2Citrum, 'W-2' citrumelo; SwiCitrum, 'Swingle' citrumelo; RangLimSourOra, 'Rangpur' lime $\times$ sour orange; US802Pumm, 'US-802' pummelo hybrid; MurTagTriT9: 'Murcott' tangor $\times$ trifoliate-9; Trif, trifoliate orange; FlyDr, 'Flying Dragon' trifoliate orange; and RangLim, 'Rangpur' lime. Species: citrandarin, Citrus reticulata $\times$ Poncirus trifoliata; citrumelo, Citrus paradisi $\times$ P. trifoliata; 'Rangpur' lime $\times$ sour orange, Citrus limonia $\times$ Citrus aurantium; 'US-802' pummelo hybrid, Citrus grandis $\times$ P. trifoliata; 'Murcott' tangor $\times$ trifoliate-9, C. reticulata $\times$ C. sinensis; trifoliate orange, P. trifoliata; 'Flying Dragon' trifoliate orange, $P$. trifoliata var. monstrosa; and 'Rangpur' lime, C. limonia. 
Table 5. Quick reference chart about the influence of 20 rootstock genotypes on canopy volume, production, productive efficiency, technological index, and industrial yield of 'Valência' sweet orange (Citrus sinensis) trees.

\begin{tabular}{|c|c|c|c|c|c|}
\hline Rootstock $^{(1)}$ & $\begin{array}{l}\text { Canopy volume } \\
\left(\mathrm{m}^{3}\right)^{(2)}\end{array}$ & $\begin{array}{c}\text { Production } \\
(\mathrm{kg} \text { per tree })^{(3)}\end{array}$ & $\begin{array}{l}\text { Productive efficiency } \\
\qquad\left(\mathrm{kg} \mathrm{m}^{-3}\right)^{(4)}\end{array}$ & $\begin{array}{l}\text { Technological index } \\
\quad(\mathrm{kg} \text { of SS per } \\
\text { box of } 40.8 \mathrm{~kg})^{(5)}\end{array}$ & $\begin{array}{c}\text { Industrial yield } \\
\text { (No. of boxes of } \\
40.8 \mathrm{~kg} \text { per ton of } \\
\text { concentrated juice) }^{(6)}\end{array}$ \\
\hline 'US-852' citrandarin & High & Optimum & Intermediate & Good & Good \\
\hline 'US-801' citrandarin & High & Optimum & Intermediate & Optimum & Optimum \\
\hline 'US-812' citrandarin & Intermediate & Good & Intermediate & Optimum & Optimum \\
\hline IPEACS-256 citrandarin & High & Optimum & Intermediate & Optimum & Optimum \\
\hline IPEACS-239 citrandarin & Low & Intermediate & Intermediate & Optimum & Optimum \\
\hline IPEACS-264 citrandarin & Very high & Optimum & Poor & Optimum & Optimum \\
\hline F.80-18 citrumelo & Low & Poor & Optimum & Good & Intermediate \\
\hline F.80-3 citrumelo & Low & Good & Good & Good & Good \\
\hline F.80-5 citrumelo & Low & Intermediate & Good & Optimum & Optimum \\
\hline F.80-6 citrumelo & Intermediate & Intermediate & Poor & Good & Good \\
\hline F.80-7 citrumelo & High & Good & Poor & Good & Intermediate \\
\hline F.80-8 citrumelo & Intermediate & Intermediate & Poor & Optimum & Optimum \\
\hline 'W-2' citrumelo & Very high & Good & Poor & Good & Good \\
\hline 'Swingle' citrumelo & Intermediate & Good & Poor & Optimum & Optimum \\
\hline 'Rangpur' lime $\times$ sour orange & Very high & Optimum & Poor & Good & Good \\
\hline 'US-802' pummelo hybrid & Intermediate & Poor & Poor & Optimum & Good \\
\hline 'Murcott' tangor $\times$ trifoliate- 9 & Intermediate & Intermediate & Intermediate & Good & Good \\
\hline Trifoliate orange & Low & Poor & Poor & Optimum & Optimum \\
\hline 'Flying Dragon' trifoliate orange & Low & Poor & Intermediate & Optimum & Optimum \\
\hline 'Rangpur' lime & Very high & Optimum & Intermediate & Good & Intermediate \\
\hline
\end{tabular}

${ }^{(1)}$ Species: citrandarin, Citrus reticulata $\times$ Poncirus trifoliata; citrumelo, Citrus paradisi $\times$ P. trifoliata; 'Rangpur' lime $\times$ sour orange, Citrus limonia $\times$ Citrus aurantium; 'US-802' pummelo hybrid, Citrus grandis $\times$ P. trifoliata; 'Murcott' tangor $\times$ trifoliate-9, C. reticulata $\times$ C. sinensis; trifoliate orange, P. trifoliata; 'Flying Dragon' trifoliate orange, P. trifoliata var. monstrosa; and 'Rangpur' lime, C. limonia. ${ }^{(2)}$ Classified as: low, $>3.5 \geq 8.0$; intermediate, $>$ $8.0<10.0$; high, $>10.0<12.0$; and very high, $>12 .{ }^{(3)}$ Classified as: optimum, $>85<105$; good, $>70<85$; intermediate, $>55<70$; and poor, $<55$. ${ }^{(4)} \mathrm{Classified}$ as: optimum, $>12$; good, $>10.0<12.0$; intermediate, $>7.5<10.0$; and poor, $<7.5$. ${ }^{(5)}$ Classified as: optimum, $\geq 2.5$; good, $>2.0<2.5$; intermediate, $>1.8$ $<2.0$; and poor, $<2.0 .{ }^{(6)}$ Classified as: optimum, $<280$; good, $>280<300$; intermediate, $>300<320$; and poor, $>300$. Classification based on means of production per tree, productive efficiency, technological index, and industrial yield in the 2016, 2017, and 2018 harvest seasons, as well as on canopy volume in the 2018 harvest season when the citrus orchard reached the mature stage.

\section{Conclusions}

1. F.80-18, F.80-5, and F.80-3 citrumelos (C. paradisi $\times P$. trifoliata) and IPEACS-239 citrandarin (C. reticulata 'Cleopatra' $\times P$. trifoliata 'Rubidoux') induce dwarf trees and a high production efficiency, being adequate rootstock options for 'Valência' sweet orange (Citrus sinensis) cultivation in the northwest of the state of Paraná, Brazil.

2. Rootstocks of F.80-5 and F.80-3 citrumelos and IPEACS-239 citrandarin are also adequate options to obtain orange juices with good industrial properties.

\section{Acknowledgments}

To Coordenação de Aperfeiçoamento de Pessoal de Nível Superior (Capes), for financing, in part, this study (Finance Code 001) by awarding a scholarship to the first author; to Dr. Jorgino Pompeu Júnior and Dr. Marines Bastianel from Centro de Citricultura Sylvio Moreira of Instituto Agronômico (CCSM/IAC), for providing the rootstock genotypes; to Dr. Kim D. Bowman from the Horticultural Research Laboratory of the Agricultural Research Service of the U.S. Department of Agriculture (USDA/ARS/HRL), for the information on the rootstocks of USDA origin; to $\mathrm{Mr}$. José Gilberto Pratinha and Mr. Ismael Lopes from the Pratinha certified citrus nursery, for the production of nursery trees; to Fernando Ortiz and Carlos Eduardo Alves da Silva from Cooperativa Nova Citrus and to Wandayr Correa from Instituto de Desenvolvimento Rural do Paraná (IDR-Paraná), for general support; all technical staff of Cocamar Cooperative for their 
constant care of the orchard in the field; and to Paulo Rizzo from Integrada Cooperativa Agroindustrial, for the physicochemical analysis of the fruits.

\section{References}

BASTOS, D.C.; FERREIRA, E.A.; PASSOS, O.S.; SÁ, J.F. de; ATAÍDE, E.M.; CALGARO, M. Cultivares copa e porta-enxertos para a citricultura brasileira. Informe Agropecuário, v.35, p.3645, 2014.

CARVALHO, L.M. de; CARVALHO, H.W.L. de; BARROS, I. de; MARTINS, C.R.; SOARES FILHO, W. dos S.; GIRARDI, E.A.; PASSOS, O.S. New scion-rootstock combinations for diversification of sweet orange orchards in tropical hardsetting soils. Scientia Horticulturae, v.243, p.169-176, 2019a. DOI: https://doi.org/10.1016/j.scienta.2018.07.032.

CARVALHO, S.A. de; GIRARDI, E.A.; MOURÃO FILHO, F. de A.A.; FERRAREZZI, R.S.; COLETTA FILHO, H.D. Advances in citrus propagation in Brazil. Revista Brasileira de Fruticultura, v.41, e-422, 2019b. DOI: https://doi.org/10.1590/010029452019422.

CASTLE, W.S.; BALDWIN, J.C.; MURARO, R.P.; LITTELL, $\mathrm{R}$. Performance of 'Valencia' sweet orange trees on 12 rootstocks at two locations and an economic interpretation as a basis for rootstock selection. HortScience, v.45, p.523-533, 2010. DOI: https://doi.org/10.21273/HORTSCI.45.4.523.

CASTLE, W.S.; TUCKER, D.P.H.; KREZDORN, A.H.; YOUTSEY, C.O. Rootstocks for Florida citrus: rootstocks selection: the first step to success. $2^{\text {nd }}$ ed. Gainesville: University of Florida, 1993. 92p.

COSTA, D.P.; STUCHI, E.S.; GIRARDI, E.A.; GESTEIRA, A. da S.; COELHO FILHO, M.A.; LEDO, C.A. da S.; FADEL, A.L.; SILVA, A.L.V. da; LEÃO, H.C. de; RAMOS, Y.C.; PASSOS, O.S.; SOARES FILHO, W. dos S. Hybrid rootstocks for Valencia sweet orange in rainfed cultivation under tropical savannah climate. Journal of Agricultural Science, v.12, p.40-55, 2020. DOI: https://doi.org/10.5539/jas.v12n11p40.

COSTA, D.P.; STUCHI, E.S.; GIRARDI, E.A.; MOREIRA, A.S.; GESTEIRA, A. da S.; COELHO FILHO, M.A.; LEDO, C.A. da S.; SILVA, A.L.V. da; LEÃO, H.C. de; PASSOS, O.S.; SOARES FILHO, W. dos S. Less is more: a hard way to get potential dwarfing hybrid rootstocks for Valencia sweet orange. Agriculture, v.11, art.354, 2021. DOI: https://doi.org/10.3390/ agriculture11040354.

DOMINGUES, A.R.; MARCOLINI, C.D.M.; GONÇALVES, C.H. da S.; GONÇALVES, L.S.A.; ROBERTO, S.R.; CARLOS, E.F. Fruit ripening development of 'Valencia' orange trees grafted on different 'Trifoliata' orange hybrid rootstocks. Horticulturae, v.7, art.3, 2021a. DOI: https://doi.org/10.3390/horticulturae7010003.

DOMINGUES, A.R.; MARCOLINI, C.D.M.; GONÇALVES, C.H. da S.; RESENDE, J.T.V. de; ROBERTO, S.R.; CARLOS, E.F. Rootstocks genotypes impact on tree development and industrial properties of 'Valencia' sweet orange juice. Horticulturae, v.7, art.141, 2021b. DOI: https://doi.org/10.3390/horticulturae7060141.
DOMINGUES, A.R.; NEVES, C.S.V.J.; YADA, I.F.U.; LEITE JUNIOR, R.P.; TAZIMA, Z.H. Performance of 'Cadenera' orange trees grafted on five rootstocks. Revista Brasileira de Fruticultura, v.40, e-764, 2018. DOI: https://doi.org/10.1590/010029452018764.

DONADIO, L.C.; LEDERMAN, I.E.; ROBERTO, S.R.; STUCHI, E.S. Dwarfing-canopy and rootstock cultivars for fruit trees. Revista Brasileira de Fruticultura, v.41, e-997, 2019. DOI: https://doi.org/10.1590/0100-29452019997.

FADEL, A.L.; STUCHI, E.S.; COUTO, H.T.Z.; RAMOS, Y.C.; MOURÃO FILHO, F. de A.A. Trifoliate hybrids as alternative rootstocks for 'Valencia' sweet orange under rainfed conditions. Scientia Horticulturae, v.235, p.397-406, 2018. DOI: https://doi.org/10.1016/j.scienta.2018.01.051.

FDACS. Florida Department of Agriculture and Consumer Services. Citrus budwood: Annual Report 2017/2018. 2018. Available at: <https://www.fdacs.gov/content/download/62638/ file/2018-annual_report_combined.pdf $>$. Accessed on: June 27 2020.

FERREIRA, D.F. Sisvar: a computer statistical analysis system. Ciência e Agrotecnologia, v.35, p.1039-1042, 2011. DOI: https://doi.org/10.1590/S1413-70542011000600001.

IDR-PARANÁ. Instituto de Desenvolvimento Rural do Paraná IAPAR-EMATER. Dados meteorológicos históricos e atuais. Available at: <http://www.idrparana.pr.gov.br/Pagina/DadosMeteorologicos-Historicos-e-Atuais>. Accessed on: Sept. 152021.

LATIMER JR., G.W. (Ed.). Official Methods of Analysis of AOAC International. 21 $1^{\text {th }}$ ed. Arlington: AOAC, 2019.

NITSCHE, P.R.; CARAMORI, P.H.; RICCE, W. da S.; PINTO, L.F.D. Atlas climático do estado do Paraná. Londrina: IAPAR, 2019.

POMPEU JUNIOR, J.; BLUMER, S. Citrumelos como portaenxertos para a laranjeira 'Valência'. Pesquisa Agropecuária Brasileira, v.46, p.105-107, 2011. DOI: https://doi.org/10.1590/ S0100-204X2011000100014.

POMPEU JUNIOR, J.; BLUMER, S. Híbridos de trifoliata como porta-enxertos para laranjeira Pêra. Pesquisa Agropecuária Tropical, v.44, p.9-14, 2014. DOI: https://doi.org/10.1590/S198340632014000100007.

R CORE TEAM. R: a language and environment for statistical computing. Vienna: R Foundation for Statistical Computing, 2019. Available at: $<$ https://www.R-project.org $>$. Accessed on: Sept. 142021.

RODRIGUES, M.J. da S.; ANDRADE NETO, R. de C.; ARAÚJO NETO, S.E. de; SOARES FILHO, W. dos S.; GIRARDI, E.A.; LESSA, L.S.; ALMEIDA, U.O. de. Performance of 'Valência' sweet orange grafted onto rootstocks in the state of Acre, Brazil. Pesquisa Agropecuária Brasileira, v.54, e01349, 2019. DOI: https://doi.org/10.1590/s1678-3921.pab2019.v54.01349.

RODRIGUES, M.J. da S.; LEDO, C.A. da S.; GIRARDI, E.A.; ALMEIDA, L.A. da H.; SOARES FILHO, W. dos S. Caracterização de frutos e propagação de portaenxertos híbridos de citros em ambiente protegido. Revista 
Brasileira de Fruticultura, v.37, p.457-470, 2015. DOI: https://doi.org/10.1590/0100-2945-068/14.

SANTOS, H.G. dos; JACOMINE, P.K.T.; ANJOS, L.H.C. dos; OLIVEIRA, V.Á. de; LUMBRERAS, J.F.; COELHO, M.R.; ALMEIDA, J.A. de; ARAÚJO FILHO, J.C. de; OLIVEIRA, J.B. de; CUNHA, T.J.F. Sistema brasileiro de classificação de solos. 5.ed. rev. e ampl. Brasília: Embrapa, 2018. 356p.

SAUNT, J. Citrus varieties of the world: an illustrated guide. $2^{\text {nd }}$ ed. Norwich: Sinclair International, 2000. 160p.

SCHINOR, E.H.; CRISTOFANI-YALY, M.; BASTIANEL, M.; MACHADO, M.A. Sunki mandarin vs. Poncirus trifoliata hybrids as rootstocks for Pera sweet orange. Journal of Agricultural
Science, v.5, p.190-200, 2013. DOI: https://doi.org/10.5539/jas. v5n6p190.

SIMONETTI, L.M.; CRISTOFANI-YALY, M.; BARROS, V.L.N.P. de; SCHINOR, E.H.; FADEL, A.L.; SOUSA, M.C.; LEONEL, S.; TECCHIO, M.A. Porta-enxertos alternativos para o cultivo de laranja 'Valência' na região sudoeste do estado de São Paulo. Citrus Research \& Technology, v.36, p.49-58, 2015. DOI: https://doi.org/10.4322/crt08916.

VASHISTH, T.; CHUN, C.; HAMPTON, M.O. Florida citrus nursery trends and strategies to enhance production of fieldtransplant ready citrus plants. Horticulturae, v.6, art.8, 2020. DOI: https://doi.org/10.3390/horticulturae6010008. 